\title{
Quantitative Serum Proteomics Reveals The Predictive and Prognostic Potential of Clusterin and Gelsolin in Head and Neck Squamous Cell Carcinoma Treated With Radiotherapy
}

\author{
Lipi Das \\ ACTREC: Advanced Centre for Treatment Research and Education in Cancer \\ Vedang Murthy \\ ACTREC: Advanced Centre for Treatment Research and Education in Cancer \\ ASHOK K VARMA ( $\square$ avarma@actrec.gov.in ) \\ ACTREC: Advanced Centre for Treatment Research and Education in Cancer https://orcid.org/0000-0001-6091-5315
}

\section{Research}

Keywords: HNSCC, differential expression, iTRAQ, parallel reaction monitoring, clusterin, gelsolin

Posted Date: August 30th, 2021

DOI: https://doi.org/10.21203/rs.3.rs-827705/v1

License: @ (i) This work is licensed under a Creative Commons Attribution 4.0 International License. Read Full License 


\section{Abstract \\ Background}

Radiotherapy (RT) with concomitant chemotherapy (CTRT) is the standard treatment for advanced head and neck squamous cell carcinoma (HNSCC). Despite advancements in treatment, a significant proportion of patients develop local recurrence and/or metastasis indicating resistance to treatment. Early identification of radio-resistant tumors using predictive and prognostic biomarkers is an important goal. We used a quantitative serum proteomics platform to evaluate the differential expression of proteins in HNSCC patients treated with CTRT.

\section{Methods}

Fifty patients with biopsy-proven, HPV-negative, squamous cell carcinoma of the oropharynx and larynx, undergoing curative CTRT were included in this prospective, IRB-approved study. Serum samples were collected before the start of RT (PreRT), 48 hours after RT (48hrsRT), and 1 week after RT (1WeekRT). Patients were classified as "good responders" or "poor responders" based on their clinical outcome at followup. Relative quantitation of serum was carried out by iTRAQ to identify differentially expressed proteins. A total of $180-200$ proteins were identified, of which twenty proteins showed more than 1.5 fold differential expression. PreRT protein expression levels were compared across good and poor responders to identify proteins with prognostic potential. Differential expression of proteins during RT was analyzed to identify proteins with predictive potential. Finally, twelve proteins were validated using targeted mass spectrometry in ten good and poor responders.

\section{Results}

A 1.5-2.5 fold pre-treatment upregulation of clusterin, gelsolin, extracellular matrix proteins, and proteins of the IGF pathway was observed in poor responders. A 2.0-5.0 fold upregulation of S100 proteins, clusterin, gelsolin, extracellular matrix proteins, IGF1, IGF2, and IGFBP3 was observed in poor responders within 48 hours to 1 week of starting RT.

\section{Conclusions}

The present results are the first report for a panel of twelve potential proteins that would help in early risk stratification and therapeutic prognosis of HNSCC treated with radiotherapy. The significant upregulation of clusterin and gelsolin at PreRT and within 48 hours to 1 week of starting RT, indicates their ability to act as prognostic and predictive markers, respectively. The panel of twelve proteins may facilitate the early identification of patients who are most likely to develop resistance to radiotherapy.

\section{Background:}

Tumors of the squamous epithelium lining the oral cavity, oropharynx, hypopharynx, nasopharynx, and larynx are categorized as head and neck squamous cell carcinoma (HNSCC) [1]. HNSCC ranks seventh in terms of incidence and sixth in mortality, worldwide [2]. Treatment of HNSCC is based on the subsite of the disease, stage of the tumor, and presence of metastases in the lymph nodes of the neck. Patients with advanced-stage III and IV tumors require aggressive treatment that involves multi-modality treatment including surgery, radiotherapy (RT), chemotherapy (CT), and chemo-radiotherapy (CTRT). This has severe side effects, such as functional impairment in swallowing and speech $[3,4]$. Despite many recent advancements in treatment, the five-year survival rate for advanced, human papillomavirus (HPV) negative HNSCC patients has remained below $60 \%[3,5]$. Metastasis, high rate of recurrence, and development of resistance to treatment serve as major deterrents for the effective treatment of $\operatorname{HNSCC}[4,6]$.

Biomarkers can be classified into three types-diagnostic, predictive, and prognostic. Diagnostic biomarkers aid in the diagnosis of the disease, whereas predictive biomarkers are used to predict the response of the patient to a treatment regime. Prognostic biomarkers are those that correlate with the progression and aggressiveness of disease and identify patients with a higher risk of relapse [7]. HNSCC is a heterogeneous cancer, which makes the identification of a universal tissue biomarker difficult [8]. The role of tissue biomarkers in the diagnosis and prognosis of HNSCC has been studied [9], and elevated levels of proteins like clusterin (CLU) and gelsolin (GSN) have been reported in malignancies of the breast, bladder, prostrate, esophagus, and larynx [10-14]. CLU is an anti-apoptotic glycoprotein known to enhance tumorigenesis, with a possible role in the development of treatment-resistant phenotype $[15,16]$. GSN is an actin-binding protein with a possible role in conferring a treatment-resistant phenotype in HNSCC and ovarian cancer $[17,18]$. However, there is little data on the use of tissue and/or circulating proteins to assess early response to RT or CTRT which could potentially help in optimizing treatment. Serum serves as a promising source for biomarker discovery studies as irrespective of the tumor site, altered metabolite and protein composition would be reflected in serum $[19,20]$. 
Mass spectrometry-based quantitative proteomics has emerged as a successful technique for global protein profiling. The sensitivity and accuracy of stable isotope labelling strategies, such as iTRAQ, coupled with its high throughput nature has made it one of the most soughtafter methods for quantitative proteome profiling. Relative quantitation is obtained as a fold change of protein abundance across two or more samples [21]. Validation is carried out by targeted quantification, where the instrument is run in the Parallel Reaction Monitoring (PRM) mode, allowing only a selected set of peptides to be scanned and quantified [22].

Considering the importance of serum protein biomarkers in the early identification of patients at risk of recurrence and relapse, a quantitative serum-based proteomics platform was used to identify differentially expressed proteins in good and poor responders during RT. Differentially expressed proteins were identified by iTRAQ to determine their prognostic and predictive potential and validated by PRM.

\section{Materials And Methods:}

\subsection{Patient data and categorization of samples:}

Blood samples were collected from 50 non-metastatic patients, with biopsy-confirmed HPV-negative oropharyngeal and laryngeal squamous cell carcinoma tumors, in an IRB-approved study. The samples were collected at different times during RT from the patients undergoing curative CTRT for locally advanced HNSCC. The first sample was collected before the start of RT and designated as PreRT. The second and third samples were collected after 48 hours and 1 week during RT and were designated 48hrsRT and 1WeekRT, respectively. The median follow-up period was 4.2 years. Patient samples were categorized based on the site of the primary tumor and favorable/unfavorable outcomes. Patients with tumors of the oropharynx formed one group and tumors of the hypopharynx and larynx formed the second group. Patients who responded well to treatment, without residual disease after RT were classified as "Good Responders", and those who did not respond to treatment, showed residual disease/recurrence, or died due to disease were classified as "Poor Responders".

\subsection{Serum isolation from blood:}

Blood samples were collected in red-capped $12.5 \mathrm{~mL}$ vacutainers, allowed to clot at $37^{\circ} \mathrm{C}$ for $15-30$ min, and centrifuged at $4500 \mathrm{rpm}$ for 10 minutes. Serum was separated in the form of a clear yellow supernatant and decanted into a fresh Eppendorf tube. Delipidation of the freshly collected serum was achieved by centrifugation at $13000 \mathrm{rpm}$ for $30 \mathrm{~min}$ and the whitish lipid layer was carefully decanted. To preserve the protein content of the delipidated serum for an extended period, a protease inhibitor (Sigma P2714) was added to each tube, mixed thoroughly, and stored at $-20^{\circ} \mathrm{C}$.

\subsection{Serum enrichment using acetonitrile precipitation:}

Serum enrichment was carried out as per the protocol standardized by our group [23]. Briefly, 10mg of serum, from a total protein concentration of $60-70 \mathrm{mg} / \mathrm{ml}$, was directly precipitated using chilled acetonitrile (ACN) in 1:1 ratio. The samples were incubated on ice for an hour, with intermittent gentle vortexing, and centrifuged at $13000 \mathrm{rpm}$ for 30-40 minutes. The supernatant was collected in a fresh Eppendorf tube and further centrifuged at $13000 \mathrm{rpm}$ for $10 \mathrm{~min}$ to remove the final traces of the precipitate. The clear supernatant was completely dried in a SpeedVac, and further re-suspended in 100-200 $\mu$ l MilliQ water. This enriched serum was quantified and a protein concentration of 1.0$2.0 \mathrm{mg} / \mathrm{ml}$ was obtained.

\subsection{In-solution reduction, alkylation, and trypsin digestion of enriched serum:}

$20 \mu \mathrm{g}$ of enriched serum was denatured using freshly prepared $6 \mathrm{M}$ urea in $50 \mathrm{mM}$ Tris $\mathrm{Cl}, \mathrm{pH}$ 8.0. This denatured serum was incubated for 1 hour at room temperature in a buffer containing 200mM DTT (Dithiothreitol, HIMEDIA MB070) in 50mM TrisCl, pH 8.0 for reduction. Alkylation was carried out by adding $200 \mathrm{mM}$ IAA (lodoacetamide, Sigma I1149) prepared in $50 \mathrm{mM} \mathrm{TrisCl,} \mathrm{pH} 8.0$ for one hour at room temperature in the dark. Unreacted IAA was quenched by adding $200 \mathrm{mM}$ DTT. Urea concentration was reduced to $\sim 0.6 \mathrm{M}^{\text {by }}$ the addition of $1 \mathrm{mM} \mathrm{CaCl}_{2}$. Trypsin was added in 1:20 (trypsin: protein) ratio and incubated overnight at $37^{\circ} \mathrm{C}$. The next day, the peptides were dried in a SpeedVac and stored at $-20^{\circ} \mathrm{C}$.

\subsection{ITRAQ labeling and strong cation exchange (SCX):}

In-solution reduction, alkylation, and trypsin digestion were carried out for enriched serum. The peptides generated were labeled using iTRAQ 4-plex labels (SCIEX 4352135). The labeled peptides were mixed in a single tube for strong cation exchange (SCX) to further fractionate the sample and to remove unlabelled peptides. The SCX cartridge was obtained from AB-SCIEX (ICAT ${ }^{\circledR}$ Cartridge - Cation Exchange-4326695). The cartridge $(200 \mu \mathrm{l}, 4.0 \mathrm{~mm} \times 15 \mathrm{~mm})$ is packed with POROS ${ }^{\circledR} 50 \mathrm{HS}$, with particle size $50 \mu \mathrm{m}$. SCX was carried out by reconstituting the labeled peptides in $2.5 \mathrm{~mL}$ of loading buffer containing $8 \mathrm{mM}$ ammonium formate, $\mathrm{pH} 3.0$. The column was conditioned with $2 \mathrm{~mL}$ loading buffer. The re-suspended labeled peptides were loaded onto the SCX column at a flow rate of $1 \mathrm{drop} / \mathrm{second}$ to allow binding of labeled peptides to the column. The column was washed with $2 \mathrm{~mL}$ loading buffer. Elution fractions were collected at $50 \mathrm{mM}, 100 \mathrm{mM}, 150 \mathrm{mM}$,

Page $3 / 12$ 
$200 \mathrm{mM}, 250 \mathrm{mM}, 300 \mathrm{mM}, 400 \mathrm{mM}$ and $500 \mathrm{mM}$ ammonium formate concentrations. Each fraction was dried, washed thrice with $0.1 \%$ formic acid, and stored at $-20^{\circ} \mathrm{C}$.

\subsection{Nano liquid chromatography-tandem mass spectrometry (nLC-MS/MS):}

In nLC-MS/MS runs, final spectra were generated for the fragment ions (MS2) having precursor ions at the detectable range of the MS1 scan. Sequence database search assigned the spectral peaks to each corresponding peptide sequence [24-26]. nLC-MS/MS IDA was carried out

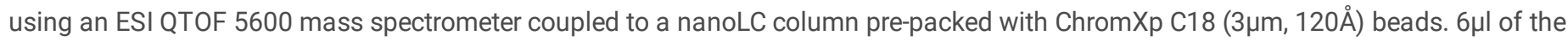
sample was injected into the column at a flow rate of $0.3 \mu \mathrm{l} / \mathrm{min}$ for a $146 \mathrm{~min}$ run using a gradient flow of solution $\mathrm{A}(0.1 \%$ formic acid in $\left.\mathrm{H}_{2} \mathrm{O}\right)$ and solution $\mathrm{B}(0.1 \%$ formic acid in $80 \% \mathrm{ACN})$. The gradient flow was as follows-initiate with $95 \%$ solution $A$ and $5 \%$ solution $B, 90 \%$ solution $A$ and $10 \%$ solution $B$ at 12 min, $70 \%$ solution A and $30 \%$ solution B at 92 min, $50 \%$ solution A and $50 \%$ solution B at 112 min, $20 \%$ solution $A$ and $80 \%$ solution $B$ at 113 min, and finally $95 \%$ solution A and 5\% solution B at 127 min and at 146 min. Data was acquired with $2.2 \mathrm{kV}$ ion spray voltage, $25 \mathrm{PSI}$ curtain gas, and $20 \mathrm{PSI}$ nebulizer gas. MS runs were operated with a resolving power (RP) of $30,000_{\mathrm{FWHM}}$. The Q1 selection range was set at 350-1250 m/z and IDA scans were acquired in $100 \mathrm{~ms}$, with $20-50$ product ion scans collected for precursor ions exceeding a threshold of 100 counts per second. Data was acquired for product ions with a +2 to +5 charge-state.

\subsection{Differential expression analysis of proteins at PreRT and during RT:}

To determine the protein expression levels in the good and poor responders before treatment is begun, enriched PreRT serum samples were labeled with iTRAQ labels, followed by nLC-MS/MS analysis. The nLC-MS/MS runs were carried out in two arms, with two sets of labels each, to eliminate any label bias. In the first arm, the good and poor responder peptide samples were labeled with 114 and 115 , respectively, whereas in the second arm, the good and poor responder samples were labeled with 116 and 117, respectively. Protein levels in good responders were considered as a baseline and the ITRAQ ratios indicated the relative expression of proteins in the poor responders.

To determine the changes in protein expression levels during RT, enriched serum of each time point was subjected to in-solution reduction, alkylation, and trypsin digestion. PreRT, 48hrsRT, and 1WeekRT peptides were labeled with 114, 115 and 116, respectively. iTRAQ data analysis was carried out by considering PreRT as a baseline and thus corresponding ratios were obtained at 48hrsRT and 1WeekRT. All iTRAQ runs were performed in biological and technical duplicates and the data presented are representative of these runs.

\subsection{Validation and relative quantitation using targeted mass spectrometry:}

Parallel reaction monitoring (PRM) using high-resolution hybrid mass spectrometers is a targeted protein quantitation method [27, 28]. It is a highly selective and specific method as a full MS/MS spectra of the targeted peptides are acquired with high resolution [27, 28]. The method involves the selection of peptide sequences that are unique to the target protein and serve as a representative for the parent protein. This parent or "precursor" ion undergoes fragmentation to produce the characteristic "product" b- and $\mathrm{y}$ - ions i.e., fragments containing the $\mathrm{N}$ - and C- termini of the peptide ions. The combination of the precursor and resulting product ions comprise "transitions" that are specific for the peptide being monitored. The peak areas for PRM transitions are integrated to measure the peptide abundance and used as the basis for quantitative comparisons [22, 29-31]. A list of 17 proteins, having at least 1.5 fold differential expression was prepared and at least two unique peptides were identified for each protein. The selected 4-25 amino acid long peptides were detected with $95 \%$ confidence in all the iTRAQ runs. The precursor m/z list was prepared using ExPasy peptide cutter (https://web.expasy.org/peptide_cutter/) and SRM Atlas (http://www.srmatlas.org/) as references. Doubly or triply charged precursors ions lying between 350-900Da were preferred for PRM analysis. The uniqueness of the selected peptides was also confirmed using the Skyline software [32]. The PRM data acquisition method was standardized in three rounds, where each round was carried out in technical duplicates. Fragment ion spectra were analyzed and at least two fragment ions were selected for each precursor $\mathrm{m} / \mathrm{z}$ for final quantitation. After the third round of standardization, precursors having $\mathrm{m} / \mathrm{z}$ greater than 900 were eliminated, as they did not ionize properly to produce clean fragment ion spectra. The retention time reproducibility was ensured in every PRM experiment and a window of $\pm 2 \mathrm{~min}$ was allowed to accommodate the biological variability of the samples. The proteins whose peptides did not yield clean fragment ion spectra were unable to be confidently quantified and removed from the validation list. The final validation list comprised of 12 proteins, with at least two peptides each. Five good responder patients and five poor responder patients were chosen at random for validation. PRM data was acquired for individual samples and correlated with the data obtained from the iTRAQ pools.

\subsection{Data analysis :}

All raw data generated by mass spectrometry were processed using PeakView software, version 1.2 (AB SCIEX). Protein identification and quantitation for iTRAQ samples were carried out using ProteinPilot software, version 4.5 (AB SCIEX). The two data sets obtained from the biological duplicates were combined and analyzed as a single dataset. The Paragon method parameters were set at $10 \%$, which corresponds to a p-value of $\mathbf{0 . 0 5}$, as the threshold for protein detection. Proteins identified with $95 \%$ confidence and $1 \%$ global FDR were considered for further analysis. To reduce variation arising due to technical parameters an additional cut-off of 1.3 fold was set [33]. Therefore proteins with

Page $4 / 12$ 
ITRAQ ratios above 1.3 and below 0.77 were identified as up- or downregulated, respectively. These proteins were submitted to the REACTOME database (https://reactome.org/) to identify the differentially regulated pathways. The possible prognostic potential of proteins was studied by comparing their expression levels in untreated good and poor responders. Protein levels were studied considering PreRT samples as control and fold changes were determined at 48hrsRT, and 1WeekRT to determine their predictive potential. Differentially expressed proteins belonging to the complement cascade and inflammatory pathways were not considered, as the expression level of these proteins may be due to altered immune response in cancer patients. Proteins belonging to the DNA damage repair pathway were also ignored as DNA damage is a characteristic side-effect of CTRT. PRM data analysis was carried out using PeakView software, version 1.2 (AB SCIEX) and MultiQuant version 2.1 (AB SCIEX).

\section{Results:}

\subsection{Patient demographics:}

Demographic details are shown in Table 1. Serum samples from 50 patients, with a median age of 53.1 years, were analysed. A majority (74.0\%) were HPV-negative oropharyngeal cancer patients. 31 patients showed excellent response to treatment and were labelled as good responders. 19 patients showed locoregional recurrence after treatment and were labelled as poor responders.

Table 1

Patient demographics

\begin{tabular}{|lll|}
\hline Category & Characteristic & Number \\
\hline Sex & Male & 50 \\
\hline Tumor site & Female & 0 \\
& Base of tongue and Vallecula & 16 \\
\cline { 2 - 3 } & Soft palate and Tonsil & 21 \\
\cline { 2 - 3 } & Hypopharynx and Larynx & 13 \\
\hline Tumor stage & T1 & 5 \\
\cline { 2 - 3 } & T2 & 29 \\
\cline { 2 - 3 } & T3 & 14 \\
\cline { 2 - 3 } & T4 & 2 \\
\hline Lymph node involvement & N0 & 29 \\
\cline { 2 - 3 } & N1 & 13 \\
\cline { 2 - 3 } & N2 & 8 \\
\hline Treatment Response & Good & 31 \\
\cline { 2 - 3 } & Poor & 19 \\
\cline { 2 - 3 } & & 29 \\
\hline
\end{tabular}

\subsection{Comparison of PreRT protein expression levels in good and poor responders to identify proteins with prognostic potential:}

Fourteen proteins involved in extracellular matrix organization and degradation, regulation and uptake of IGFs \& IGFBPs, oncogenic signalling, apoptosis, and programmed cell death were identified as being differentially expressed in poor responders (Table 2). A significant

upregulation of more than 1.5 fold was observed for clusterin, gelsolin, and SPARC in poor responders indicating their prognostic significance. The upregulation of additional proteins belonging to the IGF pathway was also observed in the laryngeal tumors, though no appreciable differences in protein levels were observed in the oropharyngeal tumors. 
Table 2

PreRT protein expression levels in poor responders. The values (indicative of the fold change) are the ITRAQ ratios obtained by considering protein expression levels in good responders as the baseline. Values greater than 1.3 indicate upregulation and values less than 0.77 indicate downregulation. Values between 1.3 and 0.77 indicate no change in protein expression levels. NQ- not quantifiable; BOT- base of tongue.

\begin{tabular}{|llllll|}
\hline Sr. No & ID & Protein & BOT/ Vallecula & Palate/ Tonsil & Hypopharynx/ Larynx \\
\hline 1 & P10909 & Clusterin & 2.65 & 1.34 & 7.60 \\
\hline 2 & P51884 & Lumican & 0.98 & 1.00 & 0.17 \\
\hline 4 & Q16610 & ECM1 & 0.99 & 0.73 & 1.00 \\
\hline 5 & P01034 & Cystatin C & 0.53 & 0.97 & 1.84 \\
\hline 6 & P06396 & Gelsolin & 1.35 & 2.25 & NQ \\
\hline 7 & P05019 & IGF1 & 1.00 & 0.96 & 3.46 \\
\hline 8 & P01344 & IGF2 & 1.42 & 1.18 & 0.27 \\
\hline 9 & P18065 & IGFBP2 & 1.05 & 0.93 & 1.04 \\
\hline 10 & P17936 & IGFBP3 & 1.01 & 1.01 & 2.61 \\
\hline 11 & P22692 & IGFBP4 & 1.00 & 0.57 & 1.72 \\
\hline 12 & P24593 & IGFBP5 & 1.36 & 1.12 & 0.52 \\
\hline 13 & P01019 & Angiotensinogen & 2.20 & NQ & 0.74 \\
\hline 14 & P04275 & von Willebrand factor & 2.11 & 0.87 & 1.70 \\
\hline
\end{tabular}

\subsection{Determining the predictive potential of proteins by analysing the differential expression of proteins during RT:}

Protein levels were studied considering PreRT samples as control and fold changes were determined at 48hrsRT and 1WeekRT (Table 3). Proteins involved in apoptosis, tumorigenesis, and cancer progression were observed to decrease by more than 1.5-fold during RT in the good responders while exhibiting a significant upregulation of more than 1.5 fold in the poor responders. The expression levels of transthyretin, galectin-3-binding protein, IGFBP4, vitronectin, and VCAM remain unchanged with RT in good responders. However, an upregulation of at least 1.5-fold is observed in the poor responders. The changes in the expression levels were observed within 48 hours to 1 week of starting RT. 
Table 3

Protein expression levels with progress of RT in good and poor responders. The values (indicative of the fold change) are the iTRAQ ratios obtained by considering protein expression levels at PreRT as the baseline. Values greater than 1.3 indicate upregulation and values less than 0.77 indicate downregulation. Values between 1.3 and 0.77 indicate no change in protein expression levels.

\begin{tabular}{|c|c|c|c|c|c|c|}
\hline \multirow[t]{2}{*}{ Sr. No } & \multirow[t]{2}{*}{ ID } & \multirow[t]{2}{*}{ Name } & \multicolumn{2}{|c|}{ GOOD RESPONDERS } & \multicolumn{2}{|c|}{ POOR RESPONDERS } \\
\hline & & & 48hrsRT & 1WeekRT & 48hrsRT & 1WeekRT \\
\hline 1 & P01019 & Angiotensinogen & 1.22 & 1.31 & 0.72 & 0.99 \\
\hline 2 & P10909 & Clusterin & 0.70 & 0.59 & 1.53 & 1.57 \\
\hline 3 & P51884 & Lumican & 1.02 & 0.72 & 1.21 & 1.67 \\
\hline 4 & P02766 & Transthyretin & 0.78 & 0.84 & 1.57 & 1.90 \\
\hline 5 & Q16610 & ECM1 & 0.70 & 0.72 & 1.47 & 1.61 \\
\hline 6 & P01034 & Cystatin-C & 0.84 & 0.66 & 1.33 & 1.35 \\
\hline 7 & Q08380 & Galectin-3 binding protein & 1.09 & 0.91 & 0.76 & 1.02 \\
\hline 8 & P06396 & Gelsolin & 0.56 & 0.51 & 1.40 & 1.50 \\
\hline 9 & P09486 & SPARC & 0.90 & 0.68 & 1.80 & 2.06 \\
\hline 10 & P05019 & IGF1 & 0.59 & 0.50 & 1.78 & 2.03 \\
\hline 11 & P01344 & IGF2 & 0.67 & 0.37 & 1.72 & 2.03 \\
\hline 12 & P17936 & IGFBP3 & 0.74 & 0.63 & 1.62 & 1.81 \\
\hline 13 & P22692 & IGFBP4 & 0.94 & 1.00 & 1.06 & 0.89 \\
\hline 14 & P04275 & von Willebrand factor & 1.12 & 1.21 & 1.24 & 1.39 \\
\hline 15 & P31151 & Protein S100-A7 & 0.63 & 0.36 & 1.43 & 1.46 \\
\hline 16 & P04004 & Vitronectin & 0.80 & 0.83 & 1.43 & 1.60 \\
\hline 17 & P19320 & VCAM & 0.80 & 1.07 & 1.95 & 2.06 \\
\hline
\end{tabular}

\subsection{Validation and relative quantitation using parallel reaction monitoring:}

An upregulation more than 1.5 fold of clusterin, lumican, extra-cellular matrix protein 1 (ECM1), cystatin C, gelsolin, SPARC, proteins of the IGF pathway, and S100A7 was observed in the poor responders, whereas a downregulation of at least 1.5 fold was observed in good responders within 48 hours of RT. PRM validation indicated a similar trend of differential regulation in individual good and poor responders (Table 4 ). An overall trend of downregulation was observed in the good responders. Though some patients did not show significant changes after 48 hours of RT, a significant downregulation of at least 1.5 fold was observed within 1 week of RT for all the good responders. In contrast, a significant upregulation of more than 1.5 fold is observed within 48 hours of RT in all the poor responders and this upregulation is maintained after 1 week of RT. 
Table 4a and 4b: PRM validation in (a) good and (b) poor responders. The values (indicative of the fold change) are the iTRAQ ratios obtained by considering protein expression levels at PreRT as the baseline. Values greater than 1.3 indicate upregulation and values less than 0.77 indicate downregulation. Values between 1.3 and 0.77 indicate no change in protein expression levels. NQ- not quantifiable.

Table 4a

\begin{tabular}{|c|c|c|c|c|c|c|c|c|c|c|c|}
\hline \multirow{2}{*}{$\begin{array}{l}\text { Sr. } \\
\text { no. }\end{array}$} & \multirow[t]{2}{*}{ Protein } & \multicolumn{2}{|l|}{ Patient 1} & \multicolumn{2}{|l|}{ Patient 2} & \multicolumn{2}{|l|}{ Patient 3} & \multicolumn{2}{|l|}{ Patient 4} & \multicolumn{2}{|l|}{ Patient 5} \\
\hline & & 48hrsRT & 1WeekRT & 48hrsRT & 1WeekRT & 48hrsRT & 1WeekRT & 48hrsRT & 1WeekRT & 48hrsRT & 1WeekRT \\
\hline 1 & Clusterin & 0.5 & 0.4 & 0.5 & 0.5 & 0.3 & 0.3 & 0.5 & 0.6 & 0.9 & 0.6 \\
\hline 2 & Gelsolin & 0.6 & 0.5 & 0.5 & 0.4 & 0.3 & 0.1 & 0.6 & 0.7 & 1.0 & 0.8 \\
\hline 3 & Lumican & 0.7 & 0.5 & 0.9 & 0.5 & 0.3 & 0.1 & 1.0 & 0.5 & 1.0 & 0.6 \\
\hline 4 & ECM1 & 0.8 & 0.6 & 0.8 & 0.3 & 0.4 & 0.1 & 0.7 & 1.1 & 1.2 & 1.1 \\
\hline 5 & $\begin{array}{l}\text { Cystatin } \\
\text { C }\end{array}$ & 0.7 & 0.7 & 0.8 & 0.6 & 0.8 & 1.0 & 0.9 & 1.0 & 1.0 & 0.9 \\
\hline 6 & SPARC & 1.2 & 1.2 & 0.6 & 0.5 & 0.5 & 0.4 & 0.8 & 0.2 & 0.9 & 0.6 \\
\hline 7 & IGF1 & 0.5 & 0.4 & 0.6 & 0.3 & 0.2 & 0.3 & 0.5 & 0.7 & 0.9 & 0.7 \\
\hline 8 & IGF2 & 1.6 & 1.2 & 0.7 & 0.4 & 0.3 & 0.5 & 0.6 & 1.0 & 0.9 & 0.8 \\
\hline 9 & IGFBP3 & 0.9 & 1.0 & 0.6 & 0.7 & 0.6 & 0.5 & 0.5 & 0.8 & NQ & NQ \\
\hline 10 & S100A7 & 0.6 & 0.3 & 0.8 & 0.4 & 0.3 & 0.3 & 0.9 & 0.5 & 0.5 & 0.3 \\
\hline 11 & S100A8 & 0.7 & 0.6 & 0.5 & 0.2 & 0.2 & 0.2 & 0.5 & 0.9 & 0.5 & 0.3 \\
\hline 12 & S100A9 & 0.6 & 0.5 & 0.8 & 0.4 & 0.1 & 0.1 & 0.3 & 0.5 & 0.9 & 0.7 \\
\hline
\end{tabular}

Table 4b

\begin{tabular}{|c|c|c|c|c|c|c|c|c|c|c|c|}
\hline \multirow{2}{*}{$\begin{array}{l}\text { Sr. } \\
\text { no. }\end{array}$} & \multirow[t]{2}{*}{ Protein } & \multicolumn{2}{|l|}{ Patient 6} & \multicolumn{2}{|l|}{ Patient 7} & \multicolumn{2}{|l|}{ Patient 8} & \multicolumn{2}{|l|}{ Patient 9} & \multicolumn{2}{|c|}{ Patient 10} \\
\hline & & 48hrsRT & 1WeekRT & 48hrsRT & 1WeekRT & 48hrsRT & 1WeekRT & 48hrsRT & 1WeekRT & 48hrsRT & 1WeekRT \\
\hline 1 & Clusterin & 3.9 & 2.4 & 3.5 & 5.6 & 3.6 & 4.1 & 2.6 & 4.3 & 1.7 & 1.9 \\
\hline 2 & Gelsolin & 3.7 & 3.5 & 4.0 & 4.8 & 2.4 & 5.7 & 3.3 & 5.8 & 1.2 & 1.2 \\
\hline 3 & Lumican & 1.4 & 2.0 & 1.7 & 2.1 & 4.5 & 4.8 & 3.3 & 3.8 & 3.2 & 5.9 \\
\hline 4 & ECM1 & 2.8 & 4.0 & 4.1 & 5.3 & 6.2 & 12.5 & 5.2 & 5.2 & 1.1 & 1.3 \\
\hline 5 & $\begin{array}{l}\text { Cystatin } \\
\text { C }\end{array}$ & 2.9 & 2.2 & 5.6 & 6.0 & 1.8 & 3.2 & 1.9 & 1.9 & 1.4 & 1.4 \\
\hline 6 & SPARC & 3.9 & 4.1 & 7.4 & 11.4 & 5.0 & 4.2 & 1.8 & 2.5 & 1.6 & 2.4 \\
\hline 7 & IGF1 & 2.4 & 3.8 & 16.4 & 27.6 & 4.8 & 5.7 & 1.4 & 2.0 & 5.5 & 1.8 \\
\hline 8 & IGF2 & 2.0 & 2.1 & 1.9 & 2.2 & 3.7 & 4.6 & 1.5 & 2.0 & 1.5 & 1.2 \\
\hline 9 & IGFBP3 & 1.3 & 0.9 & 1.7 & 2.3 & 3.2 & 4.2 & 1.8 & 2.6 & NQ & NQ \\
\hline 10 & S100A7 & 2.8 & 6.3 & 2.1 & 3.3 & 3.5 & 5.7 & 3.0 & 5.2 & 1.7 & 2.9 \\
\hline 11 & $\mathrm{~S} 100 \mathrm{~A} 8$ & 2.2 & 6.5 & 3.3 & 3.4 & 3.0 & 4.8 & 2.0 & 2.4 & 1.3 & 3.1 \\
\hline 12 & S100A9 & 2.6 & 3.4 & 4.0 & 3.6 & 2.9 & 5.3 & 4.5 & 5.8 & 2.3 & 1.7 \\
\hline
\end{tabular}

\section{Discussion:}

The high rate of locoregional recurrence and the development of resistance to treatment are major deterrents in the effective treatment of HNSCC $[4,6]$. There is a need to identify and validate protein biomarkers which can aid in the early identification of patients with higher proclivity to developing recurrence and resistance. We have identified twelve proteins which show more than 1.5 upregulation in HNSCC patients who respond poorly to CTRT, indicating their potential to act a predictive biomarkers. In addition, some of these proteins also showed a significant pre-treatment upregulation of more than 1.5 fold in poor responders, thus, indicating their potential as prognostic markers. To 
date, multiple diagnostic protein biomarkers have been reported for HNSCC such as CYFRA 21 - 1, SCCA, CEA, but none has been successfully implemented in clinical practice [9]. The consistent and significant upregulation of clusterin and gelsolin at PreRT and within 48 hours to 1 week of starting RT indicates their ability to act as early prognostic and predictive markers, respectively. The upregulation of these proteins with RT is an early indication of local failure of treatment, thus, allowing the possibility of modifying the treatment appropriately. Additionally, the pre-treatment upregulation of these proteins offers the possibility of identifying patients with the highest chance of developing recurrence before the treatment is begun.

Clusterin (CLU) is a heterodimeric, secreted glycoprotein that is expressed in a wide variety of tissues [34]. It acts as an anti-apoptotic protein by enhancing tumorigenesis which leads to the cytosolic retention of Bax (by stabilizing the Ku70-Bax complex) and interfering with Bax's proapoptotic activities [15,35]. CLU expression is upregulated in multiple solid tumors, like bladder, breast, prostate, renal, and non-small-cell lung cancer (NSCLC) [10-12,36,37]. Previous studies in esophageal squamous cell cancer (ESCC) patients indicated that the serum levels of CLU were significantly elevated and the protein may function as a tumor marker and a prognostic factor for such patients [38]. Abnormal serum CLU expression was closely associated with TNM classification of malignant tumors and lymph node metastasis, with higher CLU expression correlating with poor survival of hepatocellular carcinoma patients [39]. The protective role of CLU was observed under CTRTinduced stress which led to the overexpression of CLU, leading to the emergence of a treatment-resistant phenotype [16]. In the present study, a 3.0 fold pre-treatment upregulation of CLU in the poor responders indicated that these patients may later develop resistance to treatment or a more aggressive tumor phenotype, thus indicating a poor prognosis even before treatment is begun. Once treatment is begun, poor responders exhibit a 1.7-3.9 fold upregulation of CLU within 48 hours, with a further upregulation of 5.6 fold within 1 week of RT, providing an early indication of possible treatment failure.

In addition to CLU, a consistent upregulation of gelsolin (GSN) was also observed in the poor responders. GSN is a cytoskeleton-associated and actin-binding protein that plays a role in regulating cell morphology, motility, and apoptosis [40-42]. Significantly enhanced GSN expression was observed in oral squamous cell carcinoma (OSCC) and was associated with advanced staging [43]. Enzyme-linked immunosorbent assays (ELISA) of plasma samples indicated the potential of circulating plasma GSN as a diagnostic biomarker for head and neck cancer [14]. Immunohistochemical staining of laryngeal squamous cell carcinoma (LSCC) tissue samples indicated the association of GSN expression with poor prognosis and its potential as a novel prognostic biomarker for disease-specific and overall survival [13]. In vitro studies in human oral carcinoma cells further elucidated the role of GSN in promoting apoptosis, cell proliferation, and enhancing tumor invasion and metastasis [44]. The ability of GSN to confer chemo- and radio-resistance has been reported in NSCLC, ovarian cancer, and HNSCC, all indicating that higher levels of GSN are associated with a higher risk of developing treatment resistance [17, 18, 45, 46]. We report here, for the first time, that the levels of GSN increase by 2.4 to 4.0 fold in the worst responding patients within 48 hours, which is maintained till 1 week of RT. This upregulation may be indicative of a local failure of treatment due to acquiring a more aggressive phenotype. A pretreatment 2.0 fold upregulation of GSN further indicated its potential as an early prognostic and predictive marker for HNSCC.

Lumican, ECM1, cystatin C, and SPARC are involved in the organization of the extracellular matrix and an increase in their expression level has been reported to increased cell proliferation, migration, invasion, induction of EMT, and metastasis [47-50]. A 1.5-5.0 fold upregulation of these proteins was observed in the poor responders while downregulation of at least 2.0 fold was observed in the good responders. The insulin-like growth factor (IGF) system is involved in cell growth, differentiation, and transformation [51]. Higher levels of IGF1 and IGF2 contributed to increased differentiation and decreased apoptosis, while a high IGF1:IGFBP3 ratio in serum was shown to be indicative of a malignant HNSCC phenotype [52, 53]. Contrary to these reports, our patient cohort exhibited an overall 2.0 to 4.0 fold upregulation of IGF1, IGF2, and IGFBP3 with the progression of treatment, indicating their potential as predictive markers. The concomitant upregulation of IGFBP3 may be due to its involvement in IGF-independent pathways to regulate cancer progression. The pre-treatment levels of IGF1, IGF2, and IGFBP3 do not provide significant evidence to consider their potential as prognostic biomarkers. Proteins S100A7, S100A8, and S100A9 belong to the $\mathrm{S} 100$ family of calcium-binding proteins that regulate cellular processes such as differentiation, cell motility, migration, and maintain the tumor microenvironment [54]. A 2.0-5.0 fold upregulation in protein expression levels was observed in the poor responders within 48 hours of treatment.

The current prospective study is the first report of treatment dependent protein changes in HNSCC patients. As the study was carried out in HPV-negative patients, the pre-treatment and time dependent downregulation of CLU and GSN in the good responders indicated their potential to act as HPV-independent prognostic markers. Further validation of these proteins in a larger cohort, with healthy controls, would help to determine the sensitivity and specificity of the proteins as potential predictive and prognostic biomarkers.

\section{Conclusion:}

The consistent upregulation of CLU and GSN before treatment and the continued increase in their levels during treatment progression indicated their ability to act as early predictive and prognostic markers. The upregulation of all 12 proteins within 48 hours to 1 week of treatment indicates their usefulness in helping oncologists to identify the failure of treatment very early in the regime. Analysing the pre-

Page $9 / 12$ 
treatment levels of CLU and GSN would further allow segregation of possible good responders and poor responders and facilitate the design of a suitable treatment regime for patients at a higher risk of developing treatment resistance.

\section{Abbreviations}

Radiotherapy- RT; Chemo-radiotherapy- CTRT; head and neck squamous cell carcinoma- HNSCC; human papillomavirus- HPV; clusterin- CLU; gelsolin- GSN; parallel reaction monitoring- PRM; acetonitrile- ACN; dithiothreitol- DTT; iodoacetamide- IAA; strong cation exchange- SCX; Enzyme-linked immunosorbent assays- ELISA; oral squamous cell carcinoma- OSCC; esophageal squamous cell carcinoma- ESCC; laryngeal squamous cell carcinoma- LSCC; non-small-cell lung cancer- NSCLC; resolving power- RP.

\section{Declarations}

Ethics approval and consent to participate: The project has been approved by IEC-TMC.

\section{Consent for publication: N/A}

Availability of data and materials: The datasets used and/or analysed during the current study are available from the corresponding author on reasonable request.

Competing interests: The authors declare no conflict of interest.

Funding: Funding for this study was supported by the Terry Fox Foundation and Annual Scientific Fund from ACTREC-TMC.

Authors' contributions: LD, VM, AKV designed the experiments; LD performed the experiments; LD, VM, AKV wrote the manuscript.

Acknowledgements: Lipi Das is thankful to University Grants Commission (UGC) for fellowship. The authors thank the Proteomics and BTIS facility at ACTREC for providing necessary support to this study.

\section{References}

1. Prince A, et al. Head and neck squamous cell carcinoma: new translational therapies. Mt Sinai J Med. 2010;77(6):684-99.

2. Bray F, et al., Global cancer statistics 2018: GLOBOCAN estimates of incidence and mortality worldwide for 36 cancers in 185 countries. CA: A Cancer Journal for Clinicians, 2018. 68(6): p. 394-424.

3. Schaaij-Visser TB, et al. Protein biomarker discovery for head and neck cancer. J Proteomics. 2010;73(10):1790-803.

4. Sanderson RJ, Ironside JA. Squamous cell carcinomas of the head neck BMJ. 2002;325(7368):822-7.

5. Lee KD, Lee HS, Jeon CH. Body fluid biomarkers for early detection of head and neck squamous cell carcinomas. Anticancer Res. 2011;31(4):1161-7.

6. Argiris A, et al. Head and neck cancer. Lancet. 2008;371(9625):1695-709.

7. Mahoney KM, Atkins MB. Prognostic and predictive markers for the new immunotherapies. Oncology (Williston Park). 2014;28(Suppl 3):39-48.

8. Gleich LL, et al. Variable genetic alterations and survival in head and neck cancer. Arch Otolaryngol Head Neck Surg. 1999;125(9):94952.

9. Guerra EN, et al. Diagnostic accuracy of serum biomarkers for head and neck cancer: A systematic review and meta-analysis. Crit Rev Oncol Hematol. 2016;101:93-118.

10. Redondo M, et al. Overexpression of clusterin in human breast carcinoma. Am J Pathol. 2000;157(2):393-9.

11. Miyake $\mathrm{H}$, et al. Overexpression of clusterin in transitional cell carcinoma of the bladder is related to disease progression and recurrence. Urology. 2002;59(1):150-4.

12. Steinberg J, et al. Intracellular levels of SGP-2 (Clusterin) correlate with tumor grade in prostate cancer. Clin Cancer Res. 1997;3(10):1707-11.

13. Şahin A, et al. The promising role of Gelsolin expression to predict survival in patients with squamous cell carcinoma of the larynx. Brazilian Journal of Otorhinolaryngology; 2021.

14. Chiu CT, et al., Circulating Plasma Gelsolin: A Predictor of Favorable Clinical Outcomes in Head and Neck Cancer and Sensitive Biomarker for Early Disease Diagnosis Combined with Soluble Fas Ligand. Cancers (Basel), 2020. 12(6).

15. Zhang $\mathrm{H}$, et al. Clusterin inhibits apoptosis by interacting with activated Bax. Nat Cell Biol. 2005;7(9):909-15. 
16. Garcia-Aranda $\mathrm{M}$, et al. Clusterin inhibition mediates sensitivity to chemotherapy and radiotherapy in human cancer. Anticancer Drugs. 2017;28(7):702-16.

17. Wang PW, et al. Gelsolin regulates cisplatin sensitivity in human head-and-neck cancer. Int J Cancer. 2014;135(12):2760-9.

18. Asare-Werehene M, et al. Plasma Gelsolin Inhibits CD8(+) T-cell Function and Regulates Glutathione Production to Confer Chemoresistance in Ovarian Cancer. Cancer Res. 2020;80(18):3959-71.

19. Psychogios N, et al. The human serum metabolome. PLoS One. 2011;6(2):e16957.

20. Kaskas NM, et al. Serum biomarkers in head and neck squamous cell cancer. JAMA Otolaryngol Head Neck Surg. 2014;140(1):5-11.

21. Wasinger VC, Zeng M, Yau Y, Current status and advances in quantitative proteomic mass spectrometry. Int J Proteomics, 2013. 2013: p. 180605.

22. Liebler DC, Zimmerman LJ. Targeted quantitation of proteins by mass spectrometry. Biochemistry. 2013;52(22):3797-806.

23. Das L, Murthy V, Varma AK. Comprehensive Analysis of Low Molecular Weight Serum Proteome Enrichment for Mass Spectrometric Studies. ACS Omega. 2020;5(44):28877-88.

24. Domon B, Aebersold R. Mass spectrometry and protein analysis. Science. 2006;312(5771):212-7.

25. Kapp E, Schutz F, Overview of tandem mass spectrometry (MS/MS) database search algorithms. Curr Protoc Protein Sci, 2007. Chapter 25: p. Unit25 2.

26. Nesvizhskii Al. Protein identification by tandem mass spectrometry and sequence database searching. Methods Mol Biol. 2007;367:87119.

27. Rauniyar N. Parallel Reaction Monitoring: A Targeted Experiment Performed Using High Resolution and High Mass Accuracy Mass Spectrometry. Int J Mol Sci. 2015;16(12):28566-81.

28. Peterson AC, et al. Parallel reaction monitoring for high resolution and high mass accuracy quantitative, targeted proteomics. Mol Cell Proteomics. 2012;11(11):1475-88.

29. Desiderio DM, Kai M. Preparation of stable isotope-incorporated peptide internal standards for field desorption mass spectrometry quantification of peptides in biologic tissue. Biomed Mass Spectrom. 1983;10(8):471-9.

30. Parker $\mathrm{CE}$, Borchers $\mathrm{CH}$. Mass spectrometry based biomarker discovery, verification, and validation-quality assurance and control of protein biomarker assays. Mol Oncol. 2014;8(4):840-58.

31. Boja ES, Rodriguez H. Mass spectrometry-based targeted quantitative proteomics: achieving sensitive and reproducible detection of proteins. Proteomics. 2012;12(8):1093-110.

32. Pino LK, et al. The Skyline ecosystem: Informatics for quantitative mass spectrometry proteomics. Mass Spectrom Rev. 2020;39(3):22944.

33. Zha C, Jiang XH, Peng SF. iTRAQ-based quantitative proteomic analysis on $\mathrm{S} 100$ calcium binding protein A2 in metastasis of laryngeal cancer. PLoS One. 2015;10(4):e0122322.

34. Fritz IB, et al. Ram rete testis fluid contains a protein (clusterin) which influences cell-cell interactions in vitro. Biol Reprod. 1983;28(5):1173-88.

35. Trougakos IP, et al. Intracellular clusterin inhibits mitochondrial apoptosis by suppressing p53-activating stress signals and stabilizing the cytosolic Ku70-Bax protein complex. Clin Cancer Res. 2009;15(1):48-59.

36. Kurahashi T, et al. Expression of the secreted form of clusterin protein in renal cell carcinoma as a predictor of disease extension. BJU Int. 2005;96(6):895-9.

37. Li H, et al. Clusterin immunoexpression and its clinical significance in patients with non-small cell lung cancer. Lung. 2010;188(5):42331.

38. Guo W, et al. Serum clusterin as a tumor marker and prognostic factor for patients with esophageal cancer. Dis Markers. 2014;2014:168960.

39. Zheng W, et al. Diagnostic and prognostic significance of secretory clusterin expression in patients with hepatocellular carcinoma. Tumour Biol. 2016;37(1):999-1008.

40. Kwiatkowski DJ. Functions of gelsolin: motility, signaling, apoptosis, cancer. Curr Opin Cell Biol. 1999;11(1):103-8.

41. Kusano $\mathrm{H}$, et al. Human gelsolin prevents apoptosis by inhibiting apoptotic mitochondrial changes via closing VDAC. Oncogene. 2000;19(42):4807-14.

42. Koya RC, et al. Gelsolin inhibits apoptosis by blocking mitochondrial membrane potential loss and cytochrome c release. J Biol Chem. 2000;275(20):15343-9. 
43. Nomura $\mathrm{H}$, et al. Clinical significance of gelsolin-like actin-capping protein expression in oral carcinogenesis: an immunohistochemical study of premalignant and malignant lesions of the oral cavity. BMC Cancer. 2008;8:39.

44. Deng R, et al. Gelsolin regulates proliferation, apoptosis, migration and invasion in human oral carcinoma cells. Oncol Lett. 2015;9(5):2129-34.

45. Asare-Werehene M, et al. Pre-operative Circulating Plasma Gelsolin Predicts Residual Disease and Detects Early Stage Ovarian Cancer. Sci Rep. 2019;9(1):13924.

46. Zhao RS, et al. Gelsolin Promotes Radioresistance in Non-Small Cell Lung Cancer Cells Through Activation of Phosphoinositide 3Kinase/Akt Signaling. Technol Cancer Res Treat. 2017;16(4):512-8.

47. Nikitovic D, et al. Lumican affects tumor cell functions, tumor-ECM interactions, angiogenesis and inflammatory response. Matrix Biol. 2014;35:206-14.

48. Strojan P, et al. Serum cystatin C in patients with head and neck carcinoma. Clin Chim Acta. 2004;344(1-2):155-61.

49. Chang $\mathrm{CH}$, et al., Secreted Protein Acidic and Rich in Cysteine (SPARC) Enhances Cell Proliferation, Migration, and Epithelial Mesenchymal Transition, and SPARC Expression is Associated with Tumor Grade in Head and Neck Cancer. Int J Mol Sci, 2017. 18(7).

50. Kato Y, et al. Expression of SPARC in tongue carcinoma of stage II is associated with poor prognosis: an immunohistochemical study of 86 cases. Int J Mol Med. 2005;16(2):263-8.

51. Wu X, et al. Serum levels of insulin growth factor (IGF-I) and IGF-binding protein predict risk of second primary tumors in patients with head and neck cancer. Clin Cancer Res. 2004;10(12 Pt 1):3988-95.

52. Zhi X, et al. Expression levels of insulin-like growth factors 1 and 2 in head and neck squamous cell carcinoma. Growth Horm IGF Res. 2014;24(4):137-41.

53. Kalfert D, et al. Serum Levels of IGF-1 and IGFBP-3 in Relation to Clinical and Pathobiological Aspects of Head and Neck Squamous Cell Carcinomas. Anticancer Res. 2017;37(6):3281-6.

54. Chen H, et al. S100 protein family in human cancer. Am J Cancer Res. 2014;4(2):89-115. 